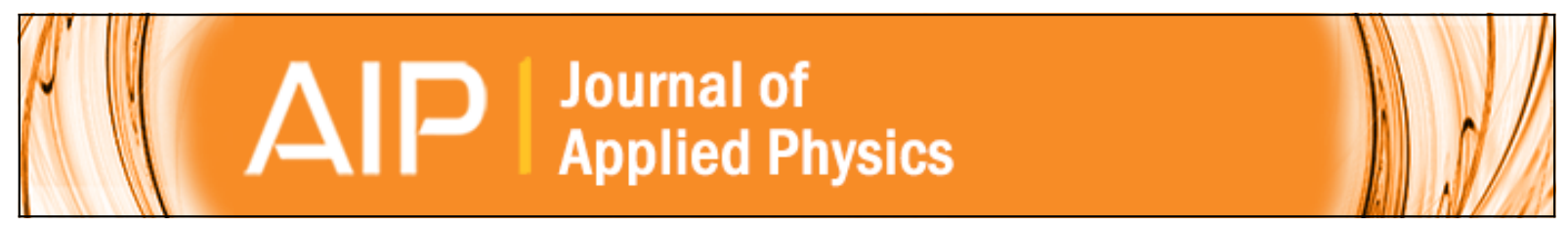

\title{
On a shock-induced martensitic phase transition
}

Rohan Abeyaratne and James K. Knowles

Citation: Journal of Applied Physics 87, 1123 (2000); doi: 10.1063/1.371989

View online: http://dx.doi.org/10.1063/1.371989

View Table of Contents: http://scitation.aip.org/content/aip/journal/jap/87/3?ver=pdfcov

Published by the AIP Publishing

\section{Articles you may be interested in}

On the Micromechanisms of ShockInduced Martensitic Transformation in Tantalum

AIP Conf. Proc. 845, 228 (2006); 10.1063/1.2263305

Electrical Conductivity Investigation of GraphiteDiamond Transition under Multiple ShockWave Compression AIP Conf. Proc. 620, 233 (2002); 10.1063/1.1483523

Transformation kinetics for the shock wave induced phase transition in cadmium sulfide crystals

J. Appl. Phys. 91, 9561 (2002); 10.1063/1.1478790

Shock-induced $\alpha-\omega$ phase transition and mechanisms of spallation in shock loaded titanium alloys

AIP Conf. Proc. 505, 439 (2000); 10.1063/1.1303511

Stress-wave-induced martensitic phase transformations in NiTi

AIP Conf. Proc. 505, 267 (2000); 10.1063/1.1303470

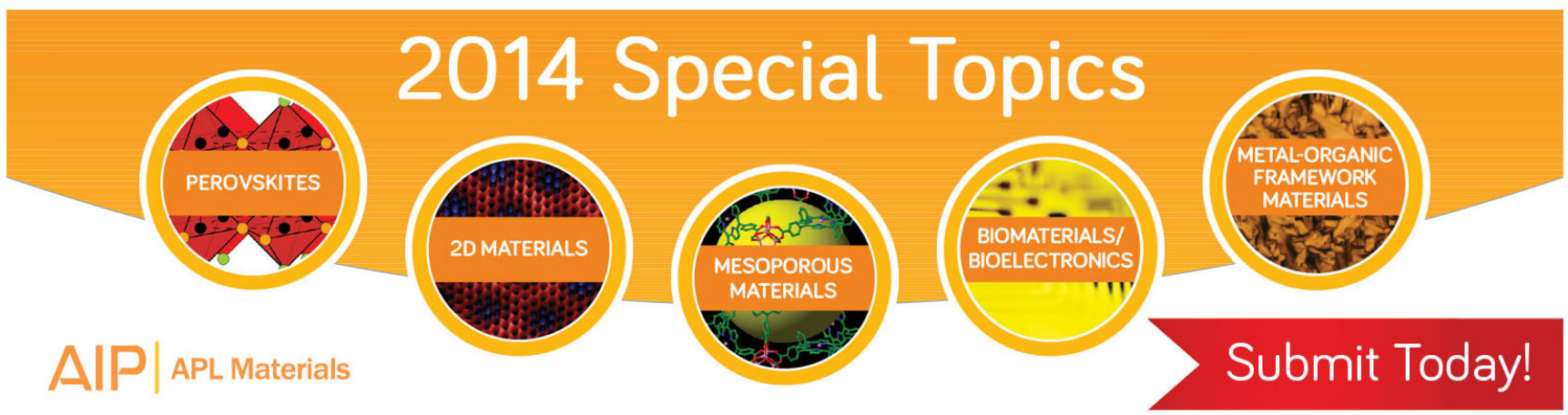




\title{
On a shock-induced martensitic phase transition
}

\author{
Rohan Abeyaratne \\ Department of Mechanical Engineering, Massachusetts Institute of Technology, \\ Cambridge, Massachusetts 02139
}

James K. Knowles ${ }^{\text {a) }}$

Division of Engineering and Applied Science, California Institute of Technology, Pasadena, California 91125

(Received 29 June 1999; accepted for publication 29 October 1999)

\begin{abstract}
A recently developed continuum-mechanical model for stress-induced phase transitions in solids is applied to a transition generated by impact. The role of transition kinetics in determining the macroscopic response to impact is discussed; in addition, the special way that "overdriven" phase boundaries emerge in this model is described. The predictions of the model are compared with experiments involving shock-induced graphite-to-diamond phase transitions. (c) 2000 American Institute of Physics. [S0021-8979(00)10503-1]
\end{abstract}

\section{INTRODUCTION}

A class of continuum models of the macroscopic response of elastic or thermoelastic solids undergoing diffusionless phase transitions has been recently developed and applied to martensitic transitions in both quasistatic and dynamic settings; see, for example Refs. $1-4$. In this article, the predictions of a model of the kind described in Ref. 4 are compared with the experimental results of Erskine and Nellis ${ }^{5}$ concerning an impact-induced graphite-to-diamond phase transition.

The experimental results reported in Ref. 5 were generated by the impact of a copper disk moving at a velocity of several kilometers per second and striking a pyrolytic graphite specimen normal to its basal plane. The specimen was backed by a lithium fluoride window through which a laser beam was reflected off the rear face of the specimen, furnishing measurements of the particle velocity on the rear face as a function of time. The graphite specimen and the LiF window are thought to have similar shock impedances as long as the specimen remains in the graphite phase, so that upon arrival of the first disturbance at the back face of the specimen, there is little reflection. Figure 1 in this article is a reproduction of Fig. 3 of Ref. 5. The figure shows four traces of particle velocity versus time arising from four different impactor velocities. There are three features of the figure of special significance here: (i) As remarked by Erskine and Nellis in their caption for the figure, the three traces corresponding to all but the highest of the impactor speeds exhibit the "two-wave structure (that) is direct evidence for a phase transition." (ii) It is also noted in Ref. 5 that the height of the first jump in particle velocity is nearly the same in all three two-wave traces. (iii) The fourth trace in the figure, associated with the largest impactor velocity, represents the overdriven condition in which the second wave has overtaken the first.

Particle-velocity time histories measured at the rear face of a specimen during impact need not exhibit the two-wave

${ }^{a)}$ Electronic mail: knowles@caltech.edu structure, even though a nonoverdriven phase transition has occurred. This appears to be the case, for example, in the experiments reported by Escobar and Clifton, ${ }^{6,7}$ in which the specimen is a single crystal of CuAlNi shape-memory alloy. In these experiments, the phase boundary travels too slowly to arrive at the rear face while measurements are being taken, so the two-wave "direct evidence" for the transition is missing. The phase transition nevertheless influences the values of particle velocity during measurement.

In the present application to the experiments in Ref. 5 of a model of the type alluded to above, emphasis will be placed on the role of transition kinetics in determining the details of the "two-wave" response to impact. The nature of overdriven shock response as predicted by the present model is also illustrated. After choosing parameters of the model to fit some of the experimental data, we calculate the particle velocities arising after the phase transition and compare them with the observed values; see Table III and Fig. 9 in Sec. VI.

For simplicity, a nonlinearly elastic model is used here, so that thermal effects are omitted; such effects are taken into account in the thermoelastic model put forward in Ref. 2.

\section{MODEL}

We idealize the graphite target, the lithium fluoride window, and the copper flyer plate in the impact experiments of Ref. 5 as homogeneous elastic materials. In the unstressed reference state, the target is taken to be a slab occupying the region $0 \leqslant x \leqslant L,-\infty<y, z<\infty$, the LiF window occupies the half-space $x \geqslant L$, and the flyer plate occupies the halfspace $x \leqslant 0$ (Fig. 2). Flyer, target, and window are assumed to remain in perfect contact throughout the time interval of interest. During the motion caused by the impact, the entire assembly is assumed to undergo uniaxial deformation, so that a particle located at the point $x, y, z$ in the undeformed state is carried at time $t$ to the point $[x+u(x, t), y, z]$, where $u(x, t)$ is the displacement in the $x$ direction. The strain and particle velocity are $\gamma=u_{x}$ and $\mathrm{v}=u_{t}$, respectively, where the subscripts indicate particle derivatives. One must have $\gamma>-1$ to assure that the mapping represented by the defor- 


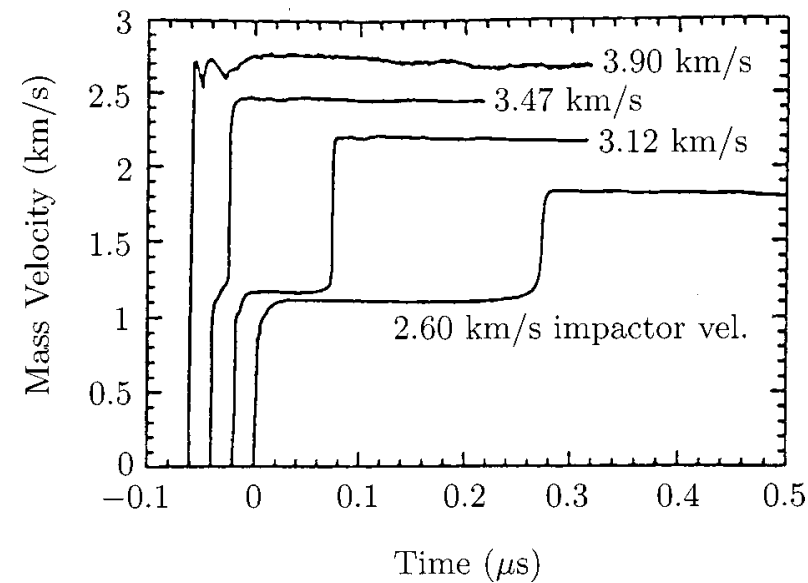

FIG. 1. Particle velocity vs time as measured in the shock experiments of Erskine and Nellis (Ref. 5). Wave profiles of pyrolytic (ZYB) graphite. The curves have been staggered horizontally on the graph for clarity. The twowave structure is direct evidence for a phase transition.

mation is one-to-one. The component of the nominal (or first Piola-Kirchhoff) stress tensor of interest here is the normal stress $\sigma(x, t)$ acting on planes perpendicular to the $x$ axis. In uniaxial deformation, there will be other nonvanishing normal stresses present as well; we assume the material symmetries of flyer, target, and window to be such that no shear stresses arise. This would be the case, for example, if the materials were isotropic, though certain forms of anisotropy would also fulfill this requirement, provided the bodies involved were suitably oriented. It should be noted that the state of stress-either nominal (Piola-Kirchhoff) or true (Cauchy) will not in general be hydrostatic in uniaxial deformation.

\section{A. Modeling the target}

We discuss first the fundamental equations appropriate to a Lagrangian description of the motion of the target; the field equations are

$$
\sigma_{x}=\rho \vee_{t},
$$

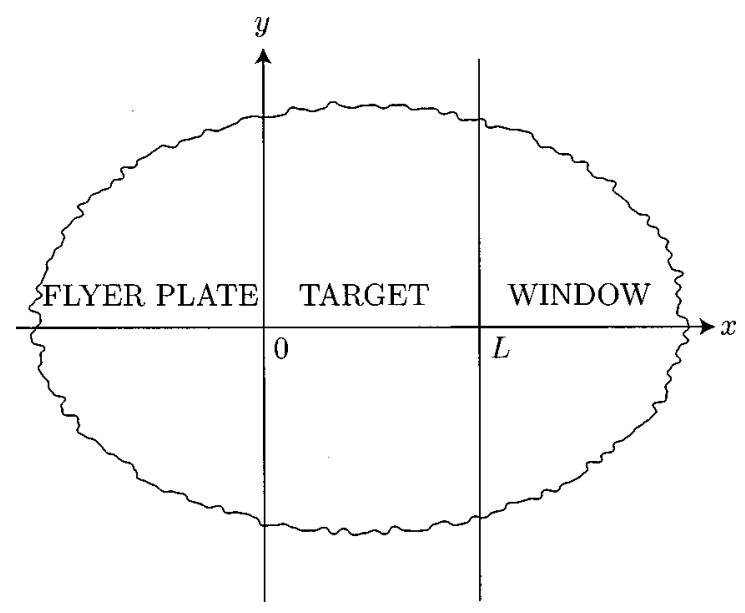

FIG. 2. Flyer plate, target and window at the instant $t=0$ of impact (Crosssection normal to $z$ axis).

$$
\begin{aligned}
& \mathrm{v}_{x}=\gamma_{t}, \\
& \sigma=\hat{\sigma}(\gamma),
\end{aligned}
$$

corresponding to balance of linear momentum, kinematic compatibility, and the bulk constitutive law, respectively; $\rho$ is the mass per unit undeformed volume of the target. In the stress-strain relation (3), the function $\hat{\sigma}(\gamma)$ is related to the strain energy per unit undeformed volume $W(\gamma)$ by $\hat{\sigma}(\gamma)$ $=W^{\prime}(\gamma)$, where the prime indicates derivative.

If there is a moving strain discontinuity located at $x$ $=s(t)$ at time $t$, the jump conditions associated with balance of momentum and kinematic compatibility are

$$
\begin{aligned}
& {[[\sigma]]=-\rho \dot{s}[[\mathrm{~V}]],} \\
& {[[\mathrm{V}]]=-\dot{s}[[\gamma]],}
\end{aligned}
$$

where, for any field quantity $\varphi(x, t)$, we have written $[[\varphi]] \equiv \varphi^{+}-\varphi^{-} \equiv \varphi[s(t)+, t]-\varphi[s(t)-, t]$. Here $\dot{s}(t)$ is the referential (or Lagrangian) velocity of the discontinuity; the "laboratory", (or Eulerian) velocity of such a discontinuity, though introduced later for comparison of the predictions of the model with experiment, will not be used in posing the impact problem, which is to be stated in fully Lagrangian form. From Eqs. (4) and (5), it may be noted that $\dot{s}$ satisfies

$$
\rho \dot{s}^{2}=\frac{\sigma^{+}-\sigma^{-}}{\gamma^{+}-\gamma^{-}} ;
$$

the right-hand side of Eq. (6) is the slope of the chord connecting the two points on the stress-strain curve that correspond to the states on either side of the discontinuity.

Next consider a portion of the target consisting of a slab of particles whose undeformed positions lie between the planes $x=x_{1}$ and $x=x_{2}$. Let

$$
D(t)=\left.\sigma \mathrm{v}\right|_{x_{1}} ^{x_{2}}-\frac{d}{d t} \int_{x_{1}}^{x_{2}}\left\{\frac{1}{2} \rho \mathrm{v}^{2}+W(\gamma)\right\} d x
$$

be the dissipation rate, defined physically as the difference between the rate of work of the forces acting on the faces of the slab and the rate of change of kinetic and stored energy, all measured per unit area normal to the $x$ axis. Suppose that the only strain discontinuity between $x_{1}$ and $x_{2}$ is at $x$ $=s(t)$. Making use of the field equations and jump conditions, one can show that

$$
D(t)=f(t) \dot{s}(t),
$$

where the driving force $f$ acting on the strain discontinuity is given by

$$
f=[[W]]-\frac{1}{2}\left(\sigma^{+}+\sigma^{-}\right)[[\gamma]] .
$$

One may interpret $f$ geometrically as the (signed) difference between the area under the stress-strain curve $\sigma=\hat{\sigma}(\gamma)$ between $\gamma^{-}$and $\gamma^{+}$and the area of the trapezoid formed by the four points $\gamma=\gamma^{-}, \sigma=0 ; \gamma=\gamma^{+}, \sigma=0 ; \gamma=\gamma^{-}, \sigma=\sigma^{-}$ $=\hat{\sigma}\left(\gamma^{-}\right)$, and $\gamma=\gamma^{+}, \sigma=\sigma^{+}=\hat{\sigma}\left(\gamma^{+}\right)$.

If there is in fact no discontinuity at $x=s(t)$, so that $[[W]]=[[\gamma]]=0$, then it follows from Eq. (9) that $f=0$, and hence from Eq. (8) that $D=0$, so that the rate of work coincides with the rate of change of total energy. On the other hand, when a genuine moving discontinuity is present, the 
driving force $f$, and therefore $D$, need not vanish. Thus, moving strain discontinuities in general produce dissipation in nonlinear elasticity theory, just as shock waves do in classical inviscid gas dynamics. In the present purely mechanical setting, the counterpart of the second law of thermodynamics is the requirement that, at any strain discontinuity, the dissipation rate $D(t)$ be nonnegative:

$$
f(t) \dot{s}(t) \geqslant 0 .
$$

Let $G(\gamma, \sigma)=W(\gamma)-\sigma \gamma$ be the potential energy per unit undeformed volume for a given stress $\sigma$. At a minimum or maximum of $G$ considered as a function of strain $\gamma$ at the fixed $\sigma$, the relation (3) necessarily holds. For the conventional linearly elastic material, Eq. (3) takes the form $\sigma$ $=\mu \gamma$, and $W(\gamma)=\mu \gamma^{2} / 2, G(\gamma, \sigma)=\mu \gamma^{2} / 2-\sigma \gamma$; thus $G$ has a single minimum at $\gamma=\sigma / \mu$. In this case, the specimen has only one equilibrium state at the given stress, corresponding to the minimum of $G$. The same is true of any nonlinearly elastic material for which $W$ is a convex function of $\gamma$. On the other hand, for an elastic material capable of existing in more than one solid phase at the stress $\sigma, G$ will have more than one minimum as a function of $\gamma$. In particular, if the material can exist in more than one phase at zero stress, $W(\gamma)$ itself will have multiple local minima, or energy wells. For a multiphase material, $W(\gamma)$ clearly cannot be convex, so that stress $\sigma=\hat{\sigma}(\gamma)=W^{\prime}(\gamma)$ cannot be a monotonic function of strain.

It may be remarked parenthetically that, in the present purely mechanical context, the value of $G$ at a local minimum is the counterpart of the Gibbs free energy per unit reference volume. If the target were in equilibrium at the stress $\sigma$, the right-hand sides of Eqs. (1) and (4) would vanish, and the stress $\sigma$ would be uniform, so that $\sigma^{+}=\sigma^{-}$at a strain discontinuity. Under this condition, the driving force $f$ of Eq. (9) would coincide with the jump in the Gibbs free energy across the discontinuity; in the dynamical setting of interest here, however, this is not the case.

For a two-phase material, $G$ as a function of $\gamma$ will have two wells at those values of $\sigma$ for which two phases exist. The simplest two-phase elastic material is a "trilinear" one of the kind exemplified in Fig. 3. The two "rising" branches of the stress-strain curve correspond to the metastable phases of the material. From the figure, for the metastable phases one has

$$
\hat{\sigma}(\gamma)=\left\{\begin{array}{l}
\mu_{0} \gamma \text { for } \gamma>-\gamma_{m}, \\
\mu_{1}\left(\gamma+\gamma_{T}\right) \text { for }-1<\gamma<-\gamma_{M} .
\end{array}\right.
$$

The material modeled by Fig. 3 changes phase in uniaxial compression; strains in the range $\gamma>-\gamma_{m}$ correspond to the low-pressure phase (LPP), while the high-pressure phase (HPP) occurs if $-1<\gamma<-\gamma_{M}$. In order to apply our model to the material studied in Eq. (5), we shall assume that the high-pressure phase is the stiffer of the two phases, so that the moduli for uniaxial deformation satisfy $\mu_{1}>\mu_{0}$. The main virtue of the trilinear model is that the nonlinear effects encountered are those associated with the phase transition itself, nonlinearities due to the bulk response of the individual phases being avoided.

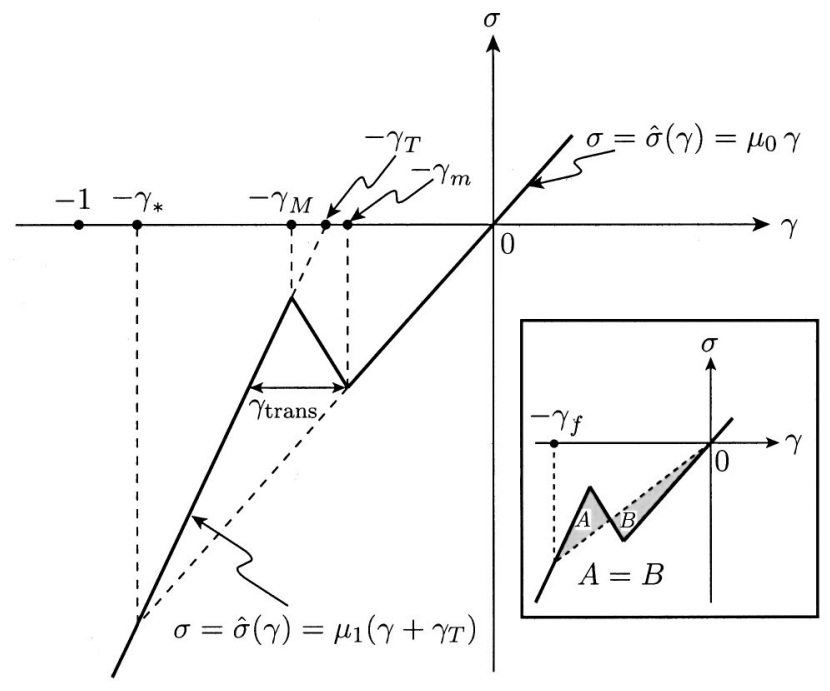

FIG. 3. Stress-strain curve for the trilinear two-phase elastic material.

There are three special strains that will arise in what follows. The first two of these are $\gamma=-\gamma_{*}$, at which the extended LPP stress-strain curve intersects its HPP counterpart, and $\gamma=-\gamma_{f}$, which, together with $\gamma=0$, cuts triangles of equal area from the stress-strain curve; see the inset in Fig. 3. The third special strain $\gamma_{\text {trans }}$ is the horizontal separation of the LPP and HPP stress-strain curves at the stress $\sigma_{m}=\hat{\sigma}\left(-\gamma_{m}\right)$ corresponding to the local minimum in Fig. 3 . These strains are given by

$$
\begin{aligned}
& \gamma_{*}=\gamma_{T} /\left(1-\mu_{0} / \mu_{1}\right), \gamma_{f}=\gamma_{m}+\gamma_{M}-\gamma_{m} \gamma_{M} / \gamma_{*}, \\
& \gamma_{\text {trans }}=\gamma_{T}-\left(1-\mu_{0} / \mu_{1}\right) \gamma_{m} .
\end{aligned}
$$

We shall require that the strain $-\gamma_{*}$ be greater than -1 ; otherwise, overdriven states could not occur in this model, as we shall see. The significance of $\gamma_{f}$ and $\gamma_{\text {trans }}$ will become clear later.

In the version of the trilinear material shown in Fig. 3, the stress $\sigma_{M}=\hat{\sigma}\left(-\gamma_{M}\right)$ at the local maximum has been assumed to be negative, so that the reference state $\gamma=0$ is the only stress-free state in which the material may find itself. If, on the other hand, $\hat{\sigma}\left(-\gamma_{M}\right)>0$, then there is also a state in the high-pressure phase at which the stress vanishes, so that the unstressed body may exist in either the low- or high-pressure phase. In the former case, upon removal of loads, the impacted body must return to the reference state, and therefore be in the low-pressure phase, whether or not a LPP-to-HPP phase transition has occurred in response to loading; thus the loading cycle is globally "reversible" in this case. In the latter situation $\left[\hat{\sigma}\left(-\gamma_{M}\right)>0\right]$, the body may remain in the high-pressure phase upon unloading if a loadinduced phase transition has taken place, in which case the cycle is globally "irreversible." In Ref. 5, evidence is cited to suggest that the graphite-to-diamond transition is globally reversible, so that the case portrayed in Fig. 3 is the appropriate one for present purposes.

For the trilinear material with $\hat{\sigma}\left(-\gamma_{M}\right)>0$, the special strain $\gamma_{T}$ has a simple interpretation: It is the magnitude of the strain jump experienced by a particle of the body when undergoing the LPP $\rightarrow \mathrm{HPP}$ transition at zero stress; it is 
therefore called the transformation strain in that case. We shall assume that $\gamma_{m}<\gamma_{T}<\gamma_{M}$, so that one has $0<\gamma_{m}$ $<\gamma_{T}<\gamma_{M}<\gamma_{f}<\gamma_{*}<1$, as indicated in Fig. 3. For the case of interest here $\left[\hat{\sigma}\left(-\gamma_{M}\right)<0\right]$, the role of transformation strain is played by $\gamma_{\text {trans }}$, as we shall show in Sec. IV.

The various requirements imposed above, together with the observation from Fig. 3 that $\hat{\sigma}\left(-\gamma_{m}\right)<\hat{\sigma}\left(-\gamma_{M}\right)$, lead to the following restriction:

$$
\left(\gamma_{M}-\gamma_{T}\right) / \gamma_{m}<\mu_{0} / \mu_{1}<1-\gamma_{T} \text {. }
$$

If the discontinuity at $x=s(t)$ involves strains $\gamma^{ \pm}$that are either both in LPP or both in HPP, we call the discontinuity a shock wave. The geometric interpretation of the driving force shows immediately that, for the trilinear material, $f$ necessarily vanishes at a shock wave in either phase, so that the entropy inequality (10) holds trivially at shocks in this model. Moreover, in this case the jump conditions (4) and (5) and the trilinear stress-strain curve lead to the conclusions that $\dot{s}=c_{0}=\sqrt{\mu_{0} / \rho}$ if $\gamma^{ \pm}$are both in LPP, while $\dot{s}$ $=c_{1}=\sqrt{\mu_{1} / \rho}$ if $\gamma^{ \pm}$are both in HPP. Thus, $c_{0}$ and $c_{1}$ are the velocities of shock waves in the two phases. For a risingfalling-rising stress-strain curve in which the metastable branches are curved, rather than straight as in Fig. 3, the speed of a shock wave is not known a priori, and the driving force at a shock does not in general vanish. Shock waves in such a more general two-phase material would therefore contribute to the dissipation, as in gas dynamics.

On the other hand, if $\gamma^{+}$and $\gamma^{-}$belong to different phases, we speak of the discontinuity as a phase boundary, and now $f$ need not vanish. In fact, at a phase boundary with HPP on the left and LPP on the right-hand side, one finds from Eq. (9) that for the trilinear material of Fig. 3, the driving force is given by

$$
f=\frac{\mu_{1}}{2}\left[-\gamma_{T} \gamma_{f}-\left(1-\mu_{0} / \mu_{1}\right) \gamma^{+} \gamma^{-}-\gamma_{T}\left(\gamma^{+}+\gamma^{-}\right)\right] .
$$

In contrast to the case of a shock wave in the trilinear material, the velocity $\dot{s}$ of a phase boundary is not determined by the jump conditions.

At the particular phase boundary for which $\gamma^{-}$is in the low-pressure phase and $\gamma^{+}=-\gamma_{*}$, the velocity $\dot{s}$ of the discontinuity is seen from Eq. (6) and Fig. 3 to coincide with the speed $c_{0}$ of LPP shock waves.

\section{B. Modeling the LiF window}

According to Ref. 5, the window and the low-pressure phase of the target have similar shock impedances. We go further by idealizing the $\mathrm{LiF}$ as a single-phase linearly elastic material whose density in the undeformed state, like that of the target, is $\rho$, and whose elastic modulus in uniaxial deformation is $\mu_{0}$, coinciding with that of the low-pressure phase of the target material. These assumptions are, of course, sufficient for impedance matching, but not necessary. The field equations and jump conditions (1) - (5) then also hold for the LiF window, with $\hat{\sigma}(\gamma)=\mu_{0} \gamma$ for all strains $\gamma>-1$. Since the stress-strain relation is linear, the driving force vanishes at a shock wave and the entropy inequality is trivially satisfied.

Because target and window are assumed to be perfectly bonded at all times of interest, both stress and particle velocity must be continuous across the target-window interface, whose undeformed location is $x=L$ :

$$
[[\sigma]]=0,[[\mathrm{v}]]=0 \text {, at } x=L .
$$

\section{Modeling the flyer plate}

We assume that the flyer plate is a homogeneous, linearly elastic material whose density in the undeformed state is $\rho_{f}$, and whose elastic modulus for uniaxial deformations is $\mu_{f}$. Differential Eqs. (1) and (2) and the jump conditions (4) and (5) now hold for $x<0$, provided $\rho$ is replaced by $\rho_{f}$; the constitutive law (3) takes the form $\sigma=\mu_{f} \gamma$. The Lagrangian speed of shock waves in uniaxial deformation is $c_{f}=\sqrt{\mu_{f} / \rho_{f}}$. It is assumed that, after impact, the flyer and the target remain in perfect contact for all times of interest here, so that stress and particle velocity are continuous across $x=0$.

To simplify the details and make the analysis as clear as possible, we shall at first assume that the flyer plate is rigid, so that $\mu_{f}=\infty$. In Sec. $\mathrm{V}$, we modify the earlier results to take the finiteness of $\mu_{f}$ into account.

\section{IMPACT PROBLEM}

In our caricature of the impact experiment described in Ref. 5, we imagine the body of Fig. 2 to be initially at rest in the reference state: $\mathrm{V}(x, 0)=\gamma(x, 0)=0$. At time $t=0$, a given constant velocity $\mathrm{v}_{0}$ is applied to the particles at the boundary $x=0$ of the target and maintained for all subsequent times, so that $\mathrm{v}(0, t)=\mathrm{v}_{0}$. Since the flyer is for now taken to be rigid, $\mathrm{v}_{0}$ coincides with the impactor velocity. For $x \geqslant 0$, $t \geqslant 0$, we wish to construct solutions to the field Eqs. (1)-(3) that satisfy the jump conditions (4) and (5) at all strain discontinuities and respect the "entropy inequality" Eq. (10) at any $\mathrm{LPP} \rightarrow$ HPP phase boundary that may arise.

\section{A. Solutions without a phase change}

For strains $\gamma>-\gamma_{m}$, target and window are composed of identical materials. Since they are perfectly bonded, for sufficiently small $\mathrm{v}_{0}$ there must be a solution of the impact problem in which there is a LPP-shock wave moving into undisturbed material, with the target remaining in the lowpressure phase behind the shock. This shock passes into the window suffering no alteration. This solution is easily seen to be given by

$$
\begin{gathered}
\gamma=\left\{\begin{array}{l}
-\mathrm{v}_{0} / c_{0} \text { for } 0 \leqslant x<c_{0} t, \\
0 \text { for } x>c_{0} t,
\end{array}\right. \\
\mathrm{v}=\left\{\begin{array}{l}
\mathrm{v}_{0} \text { for } 0 \leqslant x<c_{0} t, \\
0 \text { for } x>c_{0} t,
\end{array}\right.
\end{gathered}
$$

provided that the strain remains in the low-pressure phase, which requires that

$$
\mathrm{v}_{0}<c_{0} \gamma_{m}
$$




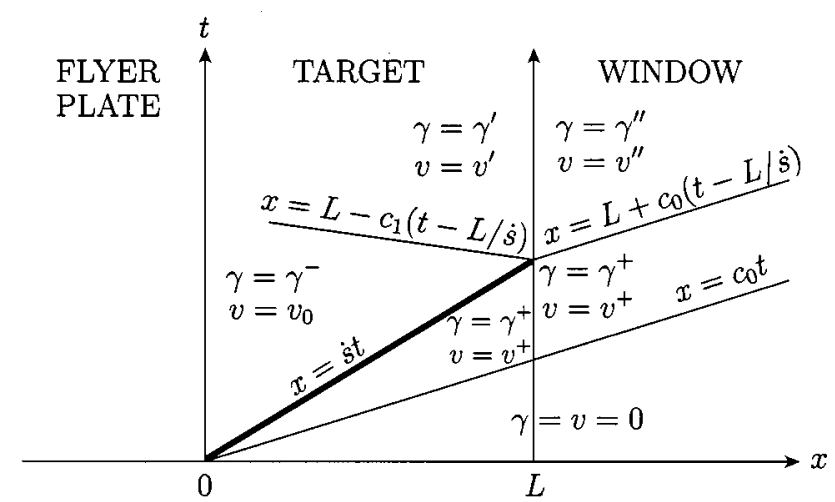

(a)

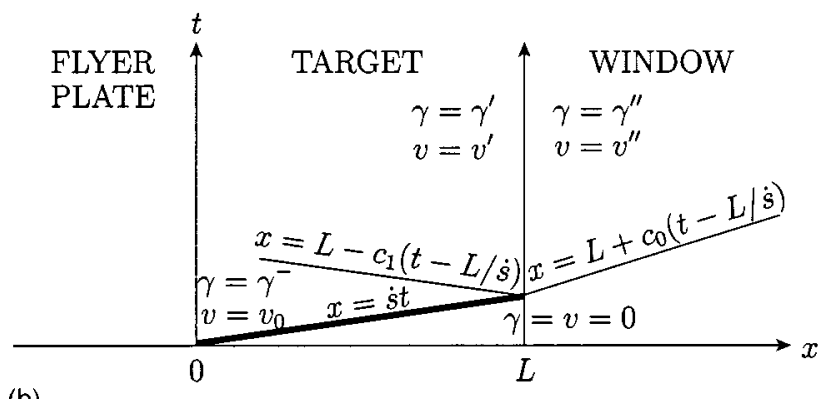

(b)

FIG. 4. Structure of solutions with phase change in the case of a rigid flyer plate. (a) Two-wave solution with phase change, (b) One-wave solution with phase change, (-) shock wave, (-) phase boundary.

\section{B. Solutions in which the target changes phase}

\section{Two-wave solutions}

There are two types of solutions corresponding to the case in which every particle of the target ultimately jumps to the high-pressure phase. In the first type [Fig. 4(a)], the target experiences a LPP-shock wave moving with speed $c_{0}$, followed by a moving phase boundary; the (Lagrangian) phase boundary velocity $\dot{s}$ must therefore be subsonic with respect to the LPP shock speed: $\dot{s}<c_{0}$. When the frontrunning shock wave strikes the target-window interface, there is no reflection because of the assumed impedance match, and the shock wave with speed $c_{0}$ continues without alteration into the $\mathrm{LiF}$ window. On the other hand, when the phase boundary arrives at this interface, the target, being in the high-pressure phase, is no longer impedance matched with the window, so a HPP shock wave with speed $c_{1}$ is reflected in the target, while a second shock wave moving with speed $c_{0}$ is initiated in the window. Assuming that the reverse transition $\mathrm{HPP} \rightarrow \mathrm{LPP}$ is not nucleated by this event, there is no "reflected phase boundary" in the target. There are, of course, infinitely many later reflections of shock waves from the two faces of the target. For sufficiently short times after impact, these later reflections do not affect the particle velocity at the target-window interface; restricting attention to such times still allows us to emulate the experiments. As indicated in Fig. 4(a), the unknowns to be determined are the constant strains $\gamma^{ \pm}, \gamma^{\prime}, \gamma^{\prime \prime}$, the constant particle velocities $\mathrm{v}^{+}, \mathrm{v}^{\prime}, \mathrm{v}^{\prime \prime}$, and the constant phase boundary velocity $\dot{s}$.
Since the unknown strains and particle velocities are all constants, the differential Eqs. (1) and (2) are trivially satisfied where $\gamma$ and $v$ are smooth. The bond conditions (15) and the jump conditions (4) and (5), applied at the various shocks and at the phase boundary [Fig. 4(a)], determine the unknown strains and particle velocities in terms of the given impactor velocity $\mathrm{v}_{0}$ and the still-unknown phase boundary velocity $\dot{s}$ as follows:

$$
\begin{aligned}
& \gamma^{-}=-\frac{1}{c_{1}^{2}+c_{0} \dot{s}}\left[\left(c_{0}+\dot{s}\right) \mathrm{v}_{0}+\gamma_{T} c_{1}^{2}\right], \\
& \gamma^{+}=-\frac{\left(c_{1}^{2}-\dot{s}^{2}\right) \mathrm{v}_{0}-\gamma_{T} c_{1}^{2} \dot{s}}{\left(c_{0}-\dot{s}\right)\left(c_{1}^{2}+c_{0} \dot{s}\right)}, \mathrm{v}^{+}=-c_{0} \gamma^{+}, \\
& \gamma^{\prime}=\frac{1}{c_{0}+c_{1}}\left[c_{0} \gamma^{-}-c_{1} \gamma_{T}-\left(c_{0} / c_{1}\right) \mathrm{v}_{0}\right], \\
& \gamma^{\prime \prime}=\frac{c_{1}}{c_{0}\left(c_{0}+c_{1}\right)}\left[c_{1}\left(\gamma^{-}+\gamma_{T}\right)-\mathrm{v}_{0}\right], \\
& \mathrm{V}^{\prime}=\mathrm{v}^{\prime \prime}=\frac{c_{1}\left(c_{1}+\dot{s}\right)}{c_{1}^{2}+c_{0} \dot{s}} \mathrm{v}_{0}-\gamma_{T} \frac{c_{0} c_{1}^{2}}{c_{0}+c_{1}} \frac{\dot{s}}{c_{1}^{2}+c_{0} \dot{s}},
\end{aligned}
$$

where $\gamma^{-}$in Eq. (19) $)_{1,2}$ is given in Eq. (18) ${ }_{1}$. In order to ensure that the target material is in the appropriate phase both behind and ahead of the phase boundary, the strains $\gamma^{+}$, $\gamma^{-}$, and $\gamma^{\prime}$ must lie in suitable intervals:

$$
-1<\gamma^{-}<-\gamma_{M}, \quad \gamma^{+}>-\gamma_{m},-1<\gamma^{\prime}<-\gamma_{M} .
$$

If $\gamma^{-}$satisfies Eq. (21) 1 , one can show with the help of Eq. $(19)_{1}$, that Eq. $(21)_{3}$ holds automatically, so that the target remains in the high-pressure phase after the passage of the reflected wave. Through Eq. (18) 1,2 , the velocities $\mathrm{v}_{0}$ and $\dot{s}$ must be subject to the restrictions imposed by the phase segregation inequalities $(21)_{1,2}$.

From Eq. (14) and (18) 1,2 one can find the driving force at the phase boundary in terms of the given impact velocity $\mathrm{v}_{0}$ and the phase boundary velocity $\dot{s}$. The result may be written in the form

$$
f=-P(\dot{s})\left(\frac{\mathrm{v}_{0}}{\gamma_{T} c_{1}}\right)^{2}+2 Q(\dot{s})\left(\frac{\mathrm{v}_{0}}{\gamma_{T} c_{1}}\right)-R(\dot{s}),
$$

where $P, Q$, and $R$ are defined by

$$
\begin{aligned}
& P(\dot{s})=\mu_{1} \frac{\gamma_{T}^{2}}{2}\left(c_{1}^{2}-c_{0}^{2}\right) \frac{c_{1}^{2}-\dot{s}^{2}}{\left(c_{1}^{2}+c_{0} \dot{s}\right)^{2}} \frac{c_{0}+\dot{s}}{c_{0}-\dot{s}}, \\
& Q(\dot{s})=\mu_{1} \frac{\gamma_{T}^{2}}{2} c_{0} c_{1} \frac{c_{1}^{2}-\dot{s}^{2}}{\left(c_{1}^{2}+c_{0} \dot{s}\right)^{2}} \frac{c_{0}+\dot{s}}{c_{0}-\dot{s}}, \\
& R(\dot{s})=\mu_{1} \frac{\gamma_{T}^{2}}{2}\left\{\frac{\gamma_{f}}{\gamma_{T}}+\frac{2 c_{0} c_{1}^{2} \dot{s}^{2}-c_{1}^{4}\left(c_{0}-\dot{s}\right)}{\left(c_{1}^{2}+c_{0} \dot{s}\right)^{2}\left(c_{0}-\dot{s}\right)}\right\} .
\end{aligned}
$$

Through Eqs. (22) - (25), a further restriction is imposed on $\mathrm{v}_{0}$ and $\dot{s}$ by the entropy inequality, which now reduces to $f$ $\geqslant 0$.

\section{One-wave solution}

The second type of solution involving a phase change has the structure shown in Fig. 4(b): The target jumps di- 
rectly from the undisturbed LPP state to the high-pressure phase without the front-running shock wave. In contrast to the case just discussed, applying the jump conditions now fully determines the unknowns $\gamma^{-}, \gamma^{\prime}, \gamma^{\prime \prime}, \mathrm{v}^{\prime}, \mathrm{v}^{\prime \prime}$, and $\dot{s}$ in terms of the given impactor velocity $\mathrm{v}_{0}$ :

$$
\begin{aligned}
& \gamma^{-}=-\frac{\gamma_{T}}{2}\left[1+\sqrt{1+4 \mathrm{v}_{0}^{2} /\left(c_{1}^{2} \gamma_{T}^{2}\right)}\right] \\
& \dot{s}=\frac{2 \mathrm{v}_{0} / \gamma_{T}}{1+\sqrt{1+4 \mathrm{v}_{0}^{2} /\left(c_{1}^{2} \gamma_{T}^{2}\right)}} . \\
& \gamma^{\prime}=\frac{1}{c_{0}+c_{1}}\left[c_{0} \gamma^{-}-c_{1} \gamma_{T}-\left(c_{0} / c_{1}\right) \mathrm{v}_{0}\right] \\
& \gamma^{\prime \prime}=\frac{c_{1}}{c_{0}\left(c_{0}+c_{1}\right)}\left[c_{1}\left(\gamma^{-}+\gamma_{T}\right)-\mathrm{v}_{0}\right] \\
& \mathrm{v}^{\prime}=\mathrm{v}^{\prime \prime}=\frac{c_{1}}{c_{0}+c_{1}}\left[\mathrm{v}_{0}+\frac{\gamma_{T}}{2} c_{1}\left(\sqrt{1+4 \mathrm{v}_{0}^{2} /\left(c_{1}^{2} \gamma_{T}^{2}\right)}-1\right)\right]
\end{aligned}
$$

In Eq. (27), $\gamma^{-}$is understood to be given by Eq. (26) ${ }_{1}$.

Through Eq. (26) $)_{1}, v_{0}$ must be restricted by the phase segregation condition $-1<\gamma^{-}<-\gamma_{M}$. If $\mathrm{v}_{0}$ fulfills this restriction, it can be shown that the further requirement -1 $<\gamma^{\prime}<-\gamma_{M}$ is automatically satisfied, so that the target remains in HPP after passage of the reflected wave.

Using the representation (14) with $\gamma^{+}=0$ and $\gamma^{-}$given by Eq. (26) ${ }_{1}$, one can find the driving force on the phase boundary for the present one-wave solution. The requirement $f \geqslant 0$ imposed by the entropy inequality becomes a further restriction on $\gamma^{-}$; this restriction turns out to be $\gamma^{-}$ $\leqslant-\gamma_{f}$, where $-\gamma_{f}$ is the special equal-area strain introduced in Eq. (12). As noted above, $\gamma^{-}$must also satisfy $-1<\gamma^{-}<-\gamma_{M}$. Since $\gamma_{f}>\gamma_{M}$, these requirements reduce to

$$
-1<\gamma^{-} \leqslant-\gamma_{f},
$$

which through Eq. (26), imposes the restriction $\sqrt{\gamma_{f}\left(\gamma_{f}-\gamma_{T}\right)} c_{1} \leqslant \mathrm{v}_{0}<\sqrt{1-\gamma_{T}} c_{1}$ on the impactor velocity.

\section{Totality of solutions}

It is helpful to catalog the solutions discussed above in a Cartesian plane in which the coordinates are the phase boundary velocity $\dot{s}$ and the impactor velocity $\mathrm{v}_{0}$, as shown in Fig. 5. We first delineate the set of points $\left(\dot{s}, \mathrm{~V}_{0}\right)$ in this plane that correspond to the no-phase-change solutions (16) permitted by the restriction (17). Thinking of these formally as special cases of the two-wave solutions of Fig. 4(a) for which $\dot{s}=0$, we may say that each no-phase-change solution corresponds to a point in the interval $0<\mathrm{v}_{0}<c_{0} \gamma_{m}$ of the vertical axis shown as bold in Fig. 5.

Next, we describe the points in the $\dot{s}, \mathrm{~V}_{0}$ plane corresponding to the two-wave solutions (18)-(20) with a phase change, subject to the phase segregation inequalities (21) and the entropy inequality $f \geqslant 0$, with $f$ given by Eqs. (22)-(25). It turns out that, of these inequalities, the decisive ones are $\gamma^{+}>-\gamma_{m}$ and $f \geqslant 0$; all others are implied by these. Using

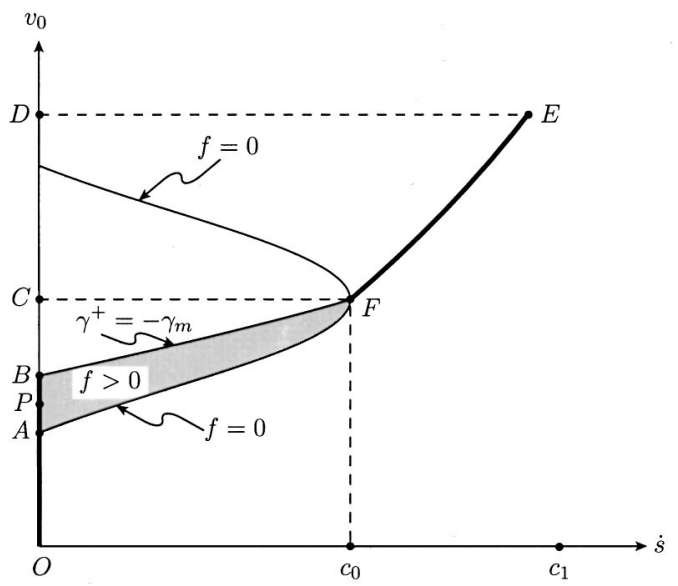

$$
\begin{array}{ll}
A: \dot{s}=0, \frac{v_{0}}{c_{1}}=\frac{\gamma_{f}-\gamma_{T}}{\frac{c_{0}}{c_{1}}+\sqrt{1-\frac{\gamma_{f}}{\gamma_{*}}}} & D: \dot{s}=0, v_{0}=\sqrt{1-\gamma_{T}} c_{1} \\
B: \dot{s}=0, v_{0}=c_{0} \gamma_{m} & E: \dot{s}=\sqrt{1-\gamma_{T}} c_{1}, v_{0}=\sqrt{1-\gamma_{T}} c_{1} \\
C: \dot{s}=0, v_{0}=\gamma_{T} c_{0} \frac{1}{1-c_{0}^{2} / c_{1}^{2}} & F: \dot{s}=c_{0}, v_{0}=\gamma_{T} c_{0} \frac{1}{1-c_{0}^{2} / c_{1}^{2}}
\end{array}
$$

FIG. 5. Pairs $\dot{s}, \mathrm{v}_{0}$ for which the impact problem has a solution. OB: solutions without a phase change. shaded region: two-wave solutions with a phase change. FE: overdriven one-wave solutions with a phase change.

Eqs. (22)-(25), one finds that $f=0$ corresponds to the parabola-like curve shown schematically in Fig. 5; for points inside this curve, one has $f>0$. The requirement $\gamma^{+}>-\gamma_{m}$ corresponds to points below the curve $\gamma^{+}=-\gamma_{m}$, also sketched schematically in Fig. 5. Thus each point in the shaded region of the figure corresponds through Eqs. (18)(20) to a two-wave solution satisfying all requirements.

Finally, the points in the $\dot{s}, \mathrm{v}_{0}$ plane that correspond to the one-wave, phase-changing solutions (26)-(28) lie on the curve represented by $(26)_{2}$, subject to the restriction (29). A portion of this curve, not shown in Fig. 5, lies inside the shaded region in Fig. 5, while the remainder lies outside this region and is shown bold as the curve FE. The points on the curve $(26)_{2}$ that lie inside the shaded area correspond to special cases of the $t w o$-wave solutions (18)-(20) in which the front-running shock happens to be absent. For this to occur, $\mathrm{V}_{0}$ and $\dot{s}$ must be suitably related; the necessary relation is precisely $(26)_{2}$. Thus only the one-wave solutions associated with points on FE correspond to solutions not already accounted for in Eqs. (18)-(20). These solutions will be seen to describe overdriven response; it may be noted from the figure that overdriven phase boundaries are supersonic with respect to the LPP shock speed $c_{0}$. As indicated in Fig. 5, the range of impactor velocities $\mathrm{v}_{0}$ for which overdriven solutions arise is the following subinterval of the interval mentioned below (29):

$$
\gamma_{T} c_{0} /\left(1-c_{0}^{2} / c_{1}^{2}\right) \leqslant \mathrm{v}_{0}<\sqrt{1-\gamma_{T}} c_{1} .
$$

The following conclusions can be drawn from Fig. 5:

(i) For each impactor velocity $v_{0}$ in the interval OA of the vertical axis, there is a unique solution given by Eq. (16); there is no phase transition.

(ii) For each $\mathrm{v}_{0}$ corresponding to a point, say $\mathrm{P}$, between $\mathrm{A}$ and $\mathrm{B}$ on the vertical axis, there are two types of solutions: 
a no-phase-change solution (16) corresponding to the point $\mathrm{P}$ itself, and a one-parameter family of two-wave solutions (18)-(20) corresponding to all points in the shaded region that lie on the horizontal line through P. Each of the latter describes a phase transition.

(iii) For each $\mathrm{v}_{0}$ that corresponds to a point on the vertical axis between $\mathrm{B}$ and $\mathrm{C}$, there is a one-parameter family of two-wave solutions (18)-(20) in which the target changes phase.

(iv) For each $v_{0}$ corresponding to a point on the vertical axis between $\mathrm{C}$ and $\mathrm{D}$, there is a unique solution; it is the one-wave solution (26)-(28) with a phase transition.

(v) For each $v_{0}$ corresponding to a point on the vertical axis above $\mathrm{D}$, there is no solution to the impact problem.

According to (i) above, unless the impact velocity is large enough, there will be no phase transition.

Conclusion (ii) shows that the impact problem for a phase-transforming nonlinearly elastic material differs drastically from its counterpart for a single-phase material: There is a massive loss of uniqueness, even with the entropy inequality in force at all strain discontinuities. For impact velocities $\mathrm{V}_{0}$ corresponding to points in the segment $\mathrm{AB}$ of the $\mathrm{V}_{0}$ axis, the target must first "choose" between the nophase-change solution (16) and the one-parameter family (parameter $\dot{s}$ ) of infinitely many two-wave, phase-changing solutions (18)-(20) that are also available for such a $\mathrm{v}_{0}$. This choice is determined by a nucleation criterion. If the choice favors the phase transition, the body must then select a particular two-wave solution from the family (18)-(20) by choosing the propagation speed $\dot{s}$. This is done through a kinetic relation.

Finally, the fact that there is no solution for values of $\mathrm{v}_{0}$ greater than $\sqrt{1-\gamma_{T}} c_{1}$, as asserted in conclusion $(\mathrm{v})$, is a consequence of the fact that such impacts result in compressive strains of magnitude greater than 1 , corresponding to infinite contraction of the specimen at a finite stress. This is an artifact of the trilinear model (Fig. 3); it would not occur for a two-phase material model in which the stress $\hat{\sigma}(\gamma)$ in the high-pressure phase tends to $-\infty$ as $\gamma$ tends to -1 .

\section{KINETICS AND NUCLEATION}

To resolve the lack of uniqueness described above, the model must be supplemented with a nucleation criterion and a kinetic relation: see, for example, Refs. 2 and 4. The nucleation criterion is expressed as a critical level of incipient driving force $f_{\text {nuc }}$ that must be achieved before the phase transition occurs. The kinetic relation, which characterizes the rate of transformation, is assumed to take the form of a connection between driving force and phase boundary velocity:

$$
f=\hat{f}(\dot{s}),-c_{0}<\dot{s}<c_{0} .
$$

For a given kinetic response function $\hat{f}(\dot{s})$, Eqs. (31) and (22) provide a relation between the impactor velocity $\mathrm{v}_{0}$ and the Lagrangian phase boundary speed $\dot{s}$ from which to find $\dot{s}$ in terms of $\mathrm{v}_{0}$.

Various forms of the function $\hat{f}$, which is considered part of the constitutive description of the material, may be derived from micromodeling such as, for example, that based on thermal activation arguments, ${ }^{1}$ or from an augmented theory in which contributions due to viscosity and straingradient are added to the elastic part of the stress, ${ }^{8}$ or from phenomenological considerations. In Eq. (31), it is usually assumed that $\hat{f}(\dot{s})$ increases monotonically with $\dot{s}$, although interesting phenomena such as stick-slip phase boundary motion are predicted when this assumption is relaxed; see Ref. 3.

As noted by Erskine and Nellis ${ }^{5}$ and supported by the data in their Table I, the particle velocities they observed after the arrival of the first shock wave in the two-wave disturbances were nearly independent of impactor velocity. If, in our model, the particle velocity $\mathrm{v}^{+}$ahead of the phase boundary in the two-wave solution is assumed to be independent of $\mathrm{v}_{0}$, then by Eq. $(18)_{3}$, the strain $\gamma^{+}$has the same property. Such a feature of the response must be due to the particular kinetics controlling the phase transition. There is a type of kinetic relation, called maximally dissipative in Ref. 9 , which has precisely this property. In this relation, the form of the function $\hat{f}$ is chosen so as to maximize the dissipation rate $D=\hat{f}(\dot{s}) \dot{s}$ for each fixed $\dot{s}$. This notion of maximum dissipation is closely related to that of maximum plastic work used in the constitutive description of rate-independent elastic-plastic solids; see, for example, Ref. 10 and 11. To determine this $\hat{f}$, one first observes that the trilinear stressstrain law and (6) yield

$$
\dot{s}^{2}=\frac{c_{0}^{2} \gamma^{+}-c_{1}^{2}\left(\gamma^{-}+\gamma_{T}\right)}{\gamma^{+}-\gamma^{-}} .
$$

Using the representation (14), one then maximizes $D$ subject to the constraint (32), $\dot{s}$ being fixed. If the fixed $\dot{s}$ is positive, as is the case in our problem, the maximizing value of the strain $\gamma^{+}$ahead of the phase boundary is found to be the strain at the local minimum in the stress-strain curve: $\gamma^{+}$ $=-\gamma_{m}$; if $\dot{s}<0$, then $D$ is maximized if $\gamma^{-}=-\gamma_{M}$, i.e., at the local maximum. From Eqs. (14) and (32), one then arrives at the kinetic relation

$$
f=\hat{f}(\dot{s})=\left\{\begin{array}{l}
\frac{\mu_{1}-\mu_{0}}{2}\left(\gamma_{*}\left(\gamma_{*}-\gamma_{f}\right)-\left(\gamma_{*}-\gamma_{m}\right)^{2} \frac{c_{0}^{2}-\dot{s}^{2}}{c_{1}^{2}-\dot{s}^{2}}\right), \\
0<\dot{s}<c_{0}, \\
\frac{\mu_{1}-\mu_{0}}{2}\left(\gamma_{*}\left(\gamma_{*}-\gamma_{f}\right)-\left(\gamma_{*}-\gamma_{M}\right)^{2} \frac{c_{1}^{2}-\dot{s}^{2}}{c_{0}^{2}-\dot{s}^{2}}\right), \\
\quad-c_{0}<\dot{s}<0 .
\end{array}\right.
$$

A schematic graph of $\hat{f}$ is shown in Fig. 6. Only the portion of the graph for which $\dot{s}>0$, corresponding to growth of HPP at the expense of LPP, is relevant in our impact problem. For completeness, however, the figure includes the portion of the kinetic relation for which $\dot{s}<0$, which controls the advance of LPP into HPP when LPP is on the right-hand side. The value $\hat{f}(0+)$ may be viewed as the resistance that must be overcome in order to cause a phase boundary to move with a positive velocity. 


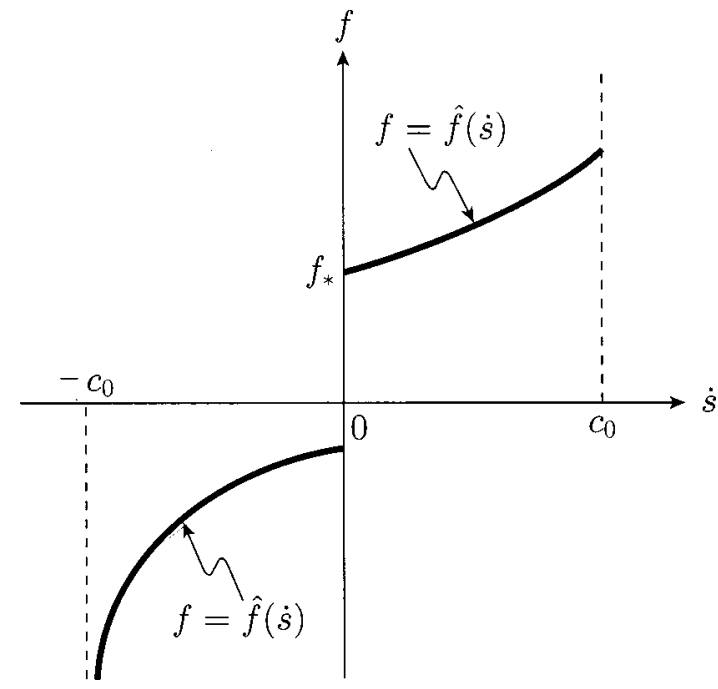

FIG. 6. Schematic graph of the maximally dissipative kinetic relation.

Kinetic relations of the form (33) that are qualitatively similar to that of Fig. 6 have been inferred in Ref. 12 from the quasistatic experiments on CuAlNi single crystals described in Ref. 13 and in 14 from impact data for the same material as reported in Refs. 6 and 7.

The nucleation site for the LPP $\rightarrow$ HPP phase transition is assumed to be at the flyer-target interface. The nucleation level $f_{\text {nuc }}$ of driving force, which must be at least as great as $\hat{f}(0+)$, is chosen here to coincide with $\hat{f}(0+)$. Upon nucleation, a phase boundary emerges at $x=0$ and moves into the target according to the evolution law (33).

Since $\gamma^{+}=-\gamma_{m}$ for maximally dissipative kinetics when $\dot{s}>0$, reference to the stress-strain curve of Fig. 3 suggests the following interpretation of the process described above: The phase change is not initiated until the given impact velocity is sufficient to drive the magnitude $|\gamma|$ of the compressive strain in the target to the largest possible value in the low-pressure phase, corresponding to the local minimum at $\gamma=-\gamma_{m}$ in Fig. 3. The phase transition then nucleates, the corresponding critical driving force being given by $f_{\text {nuc }}=\hat{f}(0+)$, which may be found from Eq. (33). Since, in this model, nucleation takes place when $\gamma^{+}=-\gamma_{m}$, the stress at nucleation is $\sigma_{\text {nuc }}=\hat{\sigma}\left(-\gamma_{m}\right)=-\mu_{0} \gamma_{m}$. Once nucleation occurs, the strain immediately jumps to a value on the high-pressure branch of the stress-strain curve, a phase boundary emerges from the impact face of the target and moves subsonically according to the kinetic law.

If the phase transition were to take place quasistatically, rather than dynamically, the right-hand side of the jump condition (4) would be replaced by zero, requiring the stress to be continuous across the phase boundary. Thus during a quasistatic transition, particles jump "horizontally" in the stress-strain diagram of Fig. 3. With nucleation occurring at $\gamma=-\gamma_{m}$ as in maximally dissipative kinetics, the magnitude of the strain jump-or quasistatic transformation strainaccompanying the phase transition would be precisely $\gamma_{\text {trans }}$, which was defined in Eq. (12).

From Eqs. (22)-(25), it is clear that the kinetic relation (33) implies a relation between the impactor velocity $\mathrm{v}_{0}$ and

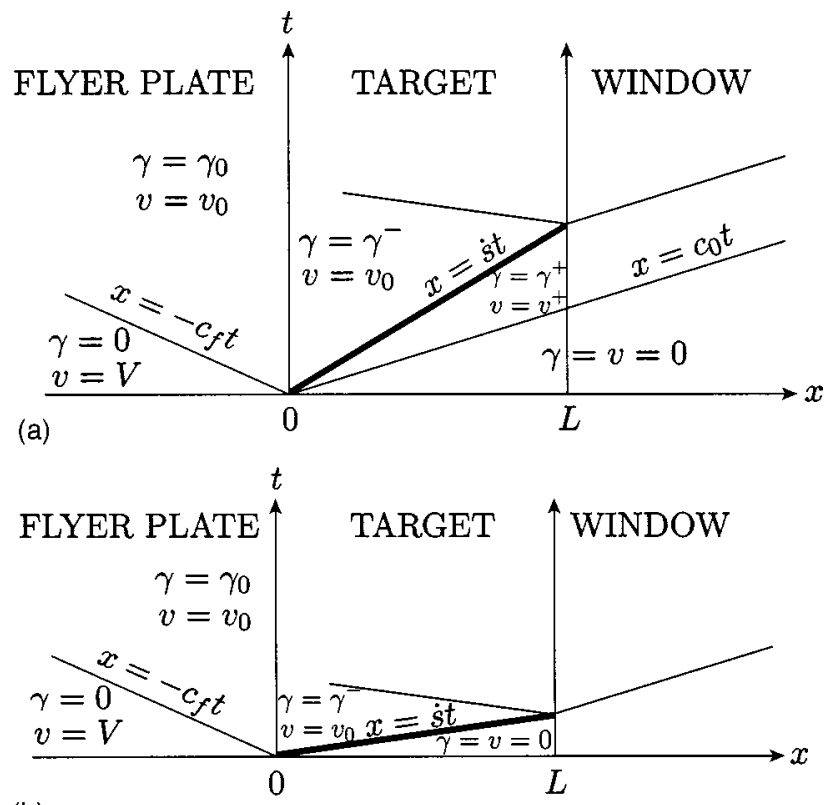

(b)

FIG. 7. Structure of solutions with phase change in the case of an elastic flyer plate. (a) Two-wave solution with phase change; (b) one-wave solution with phase change, (-) shock wave; (-) phase boundary.

the Lagrangian phase boundary velocity $\dot{s}$. For maximally dissipative kinetics with $\dot{s}>0$, this relation is most efficiently obtained by setting $\gamma^{+}=-\gamma_{m}$ in Eq. (18) $)_{2}$; the result is

$$
\begin{gathered}
\mathrm{v}_{0}=\gamma_{m} c_{0}+\left[\gamma_{T} c_{1}^{2}-\gamma_{m}\left(c_{1}^{2}-c_{0}^{2}\right)\right] \frac{\dot{s}}{c_{1}^{2}-\dot{s}^{2}}, \\
0 \leqslant \dot{s} \leqslant c_{0} \text { (two-wave solution). }
\end{gathered}
$$

For the overdriven, one-wave solution, the counterpart of Eq. (34) is $(26)_{2}$, which may be rewritten as

$$
\begin{aligned}
\mathrm{v}_{0}= & \gamma_{T} c_{1}^{2} \frac{\dot{s}}{c_{1}^{2}-\dot{s}^{2}}, \\
c_{0} & \leqslant \dot{s}<\sqrt{1-\gamma_{T} c_{1}} \text { (one-wave solution). }
\end{aligned}
$$

In the two-wave case, forms other than Eq. (34) of the $v_{0}$ $-\dot{s}$ relation would result from other choices of $\hat{f}$ in Eq. (31); there is no such choice involved in the relation (35) for the overdriven, one-wave case.

Before inquiring whether the maximally dissipative kinetic relation (33) will allow us to emulate the ErskineNellis results, we must relinquish the assumption that the flyer plate is rigid.

\section{ACCOUNTING FOR THE DEFORMABILITY OF THE FLYER PLATE}

When the modulus $\mu_{f}$ is finite, so that the flyer plate can no longer be treated as rigid, Figs. 4(a) and 4(b) describing the structure of piecewise-constant strain and particle velocity fields representing phase-changing solutions to the impact problem must be modified as shown in Figs. 7(a) and 7(b). In addition to the discontinuities generated in the target and window by impact, there is a shock wave with velocity $-c_{f}$ propagating in the flyer. The symbol $\mathrm{v}_{0}$ continues to denote 
the particle velocity at the impact face of the target, but this now differs from the impactor velocity; the latter is henceforth denoted by $V$.

By analyzing the jump conditions across the discontinuities issuing from the origin and the continuity conditions across the flyer-target interface at $x=0$ (Fig. 7), one finds in particular that $\mathrm{v}_{0}$ and $V$ are related as follows:

$$
\begin{aligned}
& \mathrm{v}_{0}=\frac{V+\frac{\gamma_{T}}{\Omega} \frac{c_{1}^{2} \dot{s}}{c_{1}^{2}+c_{0} \dot{s}}}{1+\frac{1}{\Omega} \frac{c_{1}^{2}\left(c_{0}+\dot{s}\right)}{c_{0}\left(c_{1}^{2}+c_{0} \dot{s}\right)}} \text { [two-wave case, Fig. 7(a)], } \\
& \mathrm{v}_{0}=\frac{1}{1+\dot{s} /\left(\Omega c_{0}\right)} V \text { [one-wave case, Fig. 7(b)]. }
\end{aligned}
$$

Here, $\Omega$ is the impedance ratio between flyer plate and the low-pressure phase of the target:

$$
\Omega=\frac{\rho_{f} c_{f}}{\rho c_{0}} .
$$

The rigid flyer plate corresponds to $\Omega=\infty$; in this limiting case, Eqs. (36) and (37) reduce to $\mathrm{v}_{0}=V$.

The relations between the impactor velocity $V$ and the Lagrangian phase boundary velocity $\dot{s}$ appropriate to the two types of solution are now obtained by replacing $\mathrm{v}_{0}$ in Eqs. (34) and (35) by Eqs. (36) and (37), respectively, leading ultimately to

$$
\begin{aligned}
V= & c_{0} \gamma_{m}(1+1 / \Omega)+\gamma_{\text {trans }} c_{1}^{2} \frac{\dot{s}\left[1+\dot{s} /\left(\Omega c_{0}\right)\right]}{c_{1}^{2}-\dot{s}^{2}}, \\
& 0<\dot{s} \leqslant c_{0} \quad(\text { two-wave solution), } \\
V= & \gamma_{T} c_{1}^{2} \frac{\dot{s}\left[1+\dot{s} /\left(\Omega c_{0}\right)\right]}{c_{1}^{2}-\dot{s}^{2}}, \\
& c_{0} \leqslant \dot{s}<\sqrt{1-\gamma_{T}} c_{1} \text { (one-wave solution). }
\end{aligned}
$$

The right-hand sides of both Eqs. (39) and (40) are monotonically increasing functions of $\dot{s}$ on the indicated ranges, so that both equations can be inverted to give the Lagrangian phase boundary velocity $\dot{s}$ in terms of the impactor velocity $V$. For both equations, this merely requires the solution of a quadratic equation. Once this is done, the particle velocity $\mathrm{v}_{0}$ at the flyer-target interface is found in terms of $V$ alone from Eqs. (36) or (37). The strains and particle velocities in the target can then be expressed in terms of impactor velocity from Eqs. (18)-(20) in the two-wave case, or Eqs. (26)-(28) in the one-wave case.

\section{APPLICATION OF THE MODEL TO THE EXPERIMENTS}

\section{A. Relation between Lagrangian and Eulerian phase boundary velocity}

In determining appropriate values of the material parameters for the present model from the data in Ref. 5, we need to account for the distinction between the Lagrangian and Eulerian velocities of a moving strain discontinuity. If the referential location of such a discontinuity at time $t$ is $x$ $=s(t)$, its position in the current configuration at time $t$ is $\delta(t)=s(t)+u[s(t), t]$. It follows that the respective Eulerian and Lagrangian velocities $\dot{\delta}$ and $\dot{s}$ of the discontinuity are related by

$$
\dot{\delta}=\dot{s}+\gamma^{-} \dot{s}+\mathrm{v}^{-}=\dot{s}+\gamma^{+} \dot{s}+\mathrm{V}^{+},
$$

where $\gamma^{ \pm}, \mathrm{V}^{ \pm}$are the respective strains and particle velocities on either side of the discontinuity under consideration. The consistency of the two representations in Eq. (41) is assured by the kinematic jump condition (5). If the discontinuity is the front-running shock wave in the two-wave solution (18)-(20), then Eq. (41) with $\dot{s}=c_{0}, \gamma^{+}=\mathrm{v}^{+}=0$ shows that $\dot{\delta}=c_{0}$, so that the Eulerian and Lagrangian speeds coincide, both taking the value $c_{0}$. On the other hand, if the discontinuity is the phase boundary in the two-wave solution, then for maximally dissipative kinetics one uses $\gamma^{+}$ $=-\gamma_{m}, \mathrm{v}^{+}=-c_{0} \gamma^{+}=c_{0} \gamma_{m}$ in Eq. (41) 2 . Finally, if the discontinuity is the phase boundary in the overdriven, onewave solution, then $\gamma^{+}=\mathrm{v}^{+}=0$, so $\dot{\delta}=\dot{s}$. Summarizing,

$$
\dot{\delta}=\left\{\begin{array}{l}
c_{0} \text { (front-running shock), } \\
\gamma_{m} c_{0}+\left(1-\gamma_{m}\right) \dot{s} \text { (phase boundary, } \\
\text { two-wave solution), } \\
\dot{s} \quad \text { (phase boundary, one-wave solution). }
\end{array}\right.
$$

It is the Eulerian velocity of the phase boundary that is observed in the experiments in Ref. 5.

\section{B. Material parameters}

Because some of the calculations to be described in this section and the following subsections result in a loss of numerical accuracy, we shall retain six decimal places throughout. We thus append zeros as necessary to all input data given to fewer than six decimal places. Although each material parameter found during the calculations is reported here to only three or fewer decimal places, it has been formally determined to six places, and its six-place representation is used whenever the value of the parameter is needed in subsequent calculations.

Table I of Ref. 5 reports the observed values of the velocity of the first wave (denoted by $U_{s 1}$ in Ref. 5) and the amplitude of the particle velocity ( $U_{\mathrm{pA} *}$ in Ref. 5) immediately behind this wavefront for each impactor velocity. The portion of these data that pertains to nonoverdriven response is reproduced in Table I below.

The fact that, for all impactor velocities, the speeds $U_{s 1}$ are nearly the same is consistent with the trilinear material model, for which the speed of the front-running shock is always $c_{0}$; therefore for $c_{0}$ we choose the average value of the speeds $U_{s 1}$ in the table. That all the amplitudes $U_{\mathrm{pA} *}$ in Table I are nearly the same is consistent with maximally dissipative kinetics, for which the particle velocity behind the shock is always $\gamma_{m} c_{0}$; we thus choose $\gamma_{m}$ to be such that $c_{0} \gamma_{m}$ takes the average value of the amplitudes $U_{\mathrm{pA}} *$. These choices give

$$
\gamma_{m}=0.168, c_{0}=6.962 \mathrm{~km} / \mathrm{s} \text {. }
$$


TABLE I. Experimental values of speed and amplitude of nonoverdriven first wave for various impactor velocities. ${ }^{\text {a }}$ (All velocities in kilometers per second.)

\begin{tabular}{ccc}
\hline \hline $\begin{array}{c}\text { Impactor } \\
\text { velocity } V\end{array}$ & $\begin{array}{c}\text { Speed } U_{s 1} \\
\text { of first wave }\end{array}$ & $\begin{array}{c}\text { Amplitude } U_{p A *} \\
\text { of particle velocity } \\
\text { after first wave }\end{array}$ \\
\hline 2.602 & 6.961 & 1.181 \\
2.603 & 6.873 & 1.110 \\
2.605 & 6.931 & 1.164 \\
3.120 & 6.966 & 1.173 \\
3.471 & 7.077 & 1.223 \\
\hline \hline
\end{tabular}

${ }^{\mathrm{a}}$ See Ref. 5.

For the referential density $\rho$, we choose the average value of the initial densities of the specimens reported in Table I of Ref. 5; this gives

$$
\rho=2.256 \mathrm{gm} / \mathrm{cm}^{3} \text {. }
$$

To determine the value of $\Omega$ from Eq. (38), we require the values of $c_{f}$ and $\rho_{f}$ for the copper flyer plate. Since the flyer is being treated as linearly elastic, and since it is assumed to be in a state of uniaxial deformation, the speed $c_{f}$ in Eq. (38) is the velocity of longitudinal waves in copper: this is given by Marsh ${ }^{15}$ as $c_{f}=4.76 \mathrm{~km} / \mathrm{s}$. Marsh also gives the density as $\rho_{f}=8.92 \mathrm{gm} / \mathrm{cm}^{3}$. Using these values of $c_{f}, \rho_{f}$ and the values in Eqs. $(43)_{2}$ and (44) for $c_{0}$ and $\rho$, one finds that

$$
\Omega=2.704 \text {. }
$$

Measured values of the velocity of the second wave, which is denoted by $U_{s 2}$ in Ref. 5, are also listed in Table I of Ref. 5; we reproduce these in our Table II. (In a footnote to their Table I, Erskine and Nellis state that the experiment involving the greatest impactor velocity resulted in incomplete data; in this article, we make no use of any information pertaining to this shot, which is labled "gi'" in Ref. 5.)

We now use some of the information in this table to determine $\gamma_{T}$ and $c_{1}$. Noting that the first three shots listed in Table II differ little in impact velocity, we replace them for purposes of finding $\gamma_{T}, c_{1}$ by an "average shot" whose impactor velocity is the average $\bar{V}=2.603333$ of the three lowest impactor velocities in the experiments, and whose associated second-wave speed is the average $\bar{U}_{s 2}$ $=4.781667$ of the corresponding three observed speeds. We then determine the two constants $\gamma_{T}$ and $c_{1}$ by fitting the

TABLE II. Experimental values of speed of second wave for various impactor velocities. ${ }^{a}$ (All velocities in kilometers per second.)

\begin{tabular}{cc}
\hline \hline $\begin{array}{c}\text { Impactor } \\
\text { velocity } V\end{array}$ & $\begin{array}{c}\text { Speed } U_{s 2} \\
\text { of second wave }\end{array}$ \\
\hline 2.602 & 4.727 \\
2.603 & 4.859 \\
2.605 & 4.759 \\
3.120 & 6.164 \\
3.471 & 6.922 \\
$3.900^{\mathrm{b}}$ & $7.660^{\mathrm{b}}$ \\
\hline \hline
\end{tabular}

${ }^{a}$ See Ref. 5.

${ }^{\mathrm{b}}$ Overdriven.

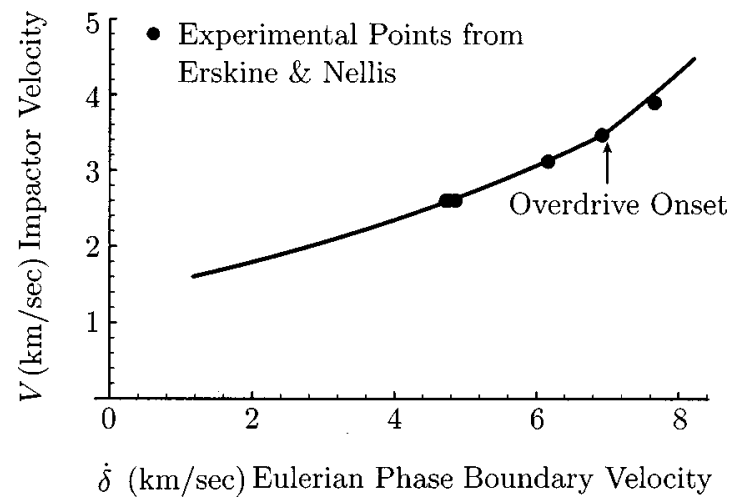

FIG. 8. Relation between impactor velocity $V$ and phase boundary velocity $\dot{\delta}$ as determined by the maximally dissipative kinetic relation. Comparison with experimental results from Ref. 5.

two-wave relation (39) between $V$ and $\dot{s}$ to the data consisting of the "average" pair $(2.603333,4.781667)$ and the pair $(3.471000,6.922000)$ that corresponds to the fastest impact experiment with nonoverdriven response. To do this, we must first use Eq. (42) $)_{2}$ to find the Lagrangian second-wave speeds $\dot{s}$ associated with these two data pairs. This process leads to two equations for $\gamma_{T}, c_{1}$. To three decimal places, these yield

$$
\gamma_{T}=0.335, c_{1}=23.908 \mathrm{~km} / \mathrm{s} \text {. }
$$

Figure 8 shows the graph of the relation between impactor velocity $V$ and Eulerian phase boundary velocity $\dot{\delta}$ provided by Eqs. (39), (40), and (42) 2,3 , using the values of the material constants $\gamma_{m}, c_{0}, \Omega, c_{1}$, and $\gamma_{T}$ just determined. The portion of the curve to the right of the arrow consists of overdriven states; where the overdriven and nonoverdriven portions of the curve meet, there is a kink. The figure also shows the six experimental pairs $\left(U_{s 2}, V\right)$ that correspond to the entries in Table II.

From the six decimal-place versions of Eqs. (43) 2 , (44), and $(46)_{2}$, we find the moduli for uniaxial deformation in the two phases:

$$
\mu_{0}=\rho c_{0}^{2}=109.3 \mathrm{GPa}, \mu_{1}=\rho c_{1}^{2}=1289.3 \mathrm{GPa} .
$$

(It is to be emphasized that these moduli are neither Young's moduli, which are relevant for uniaxial stress, nor bulk moduli, which apply for hydrostatic stress in isotropic materials.) The values of $\gamma_{m}, \gamma_{T}, \mu_{0}$, and $\mu_{1}$ just determined are such that the right member of the inequality (13) is satisfied; the left member imposes the restriction $\gamma_{M}<0.349$ on the material constant $\gamma_{M}$, which is left undetermined by the fitting process described above.

\section{Predictions of the model}

In Fig. 8, the agreement between the curve furnished by the model and the experimental points corresponding to the five nonoverdriven cases was virtually forced by the fitting process just described. One might, however, view the overdriven portion of the curve as a prediction of the model, since the overdriven data were not used in the fitting process. For the impactor velocity $V=3.900 \mathrm{~km} / \mathrm{s}$ of the overdriven 
TABLE III. Particle velocity at target-window interface after passage of the phase boundary, and phase boundary velocity. From model: $\mathrm{v}^{\prime}, \delta$. From experiments: ${ }^{\mathrm{a}} U_{p B^{*}}, U_{s 2}$. (All velocities in kilometers per second.)

\begin{tabular}{cllll}
\hline \hline$V$ & $\mathrm{v}^{\prime}$ & $U_{p B^{*}}$ & $\dot{\delta}$ & $U_{s 2}$ \\
\hline 2.602 & 1.913 & 1.850 & 4.778 & 4.727 \\
2.603 & 1.914 & 1.830 & 4.781 & 4.859 \\
2.605 & 1.915 & 1.812 & 4.786 & 4.759 \\
3.120 & 2.285 & 2.202 & 6.133 & 6.164 \\
3.471 & 2.534 & 2.460 & 6.922 & 6.922 \\
$3.900^{\mathrm{b}}$ & $2.837^{\mathrm{b}}$ & $2.704^{\mathrm{b}}$ & $7.505^{\mathrm{b}}$ & $7.660^{\mathrm{b}}$ \\
\hline \hline
\end{tabular}

${ }^{a}$ See Ref. 5.

${ }^{\mathrm{b}}$ Overdriven.

experiment, the value of phase boundary velocity predicted by the model is $\delta=7.505 \mathrm{~km} / \mathrm{s}$, while the observed value is $U_{s 2}=7.660 \mathrm{~km} / \mathrm{s}$; the error is about $2 \%$ of the observed value.

The particle velocity at the target-window interface is given by

$\mathrm{V}(L, t)$

$$
=\left\{\begin{array}{l}
0, \quad 0 \leqslant t<L / c_{0}, \\
\gamma_{m} c_{0}, \quad L / c_{0}<t<L / \dot{s}, \quad \text { (two-wave solution } \\
\mathrm{v}^{\prime}, \quad t>L / \dot{s},
\end{array}\right.
$$

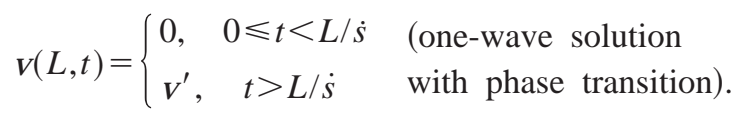

In Eq. (48), the particle velocity after the arrival of the frontrunning shock until the arrival of the phase boundary is given by $\gamma_{m} c_{0}$ because of the maximally dissipative kinetics; the particle velocity $\mathrm{v}^{\prime}$ after the phase transition is given by Eq. (20), in which $\mathrm{v}_{0}$ is to be expressed in terms of $V$ and $\dot{s}$ by Eq. (36), and $\dot{s}$ is to be found in terms of the impactor velocity $V$ by inverting Eq. (39). In Eq. (49), $v^{\prime}$ is given by Eq. (28), with $\mathrm{V}_{0}$ in turn given by Eq. (35), and with $\dot{s}$ expressed in terms of $V$ through the inversion of Eq. (40). Table III lists the resulting values of $v^{\prime}$ predicted by the model for the various impactor velocities, along with the corresponding experimentally observed values $U_{\mathrm{pB}} *$. The table also includes the predicted numerical values of the Eulerian phase boundary velocity $\dot{\delta}$, and the corresponding experimental values $U_{s 2}$ reported in Ref. 5 .

The maximum per cent error in approximating $U_{\mathrm{pB}} *$ by $\mathrm{V}^{\prime}$ is $5.7 \%$ of the observed value; it occurs for the shot with impactor velocity $V=2.605 \mathrm{~km} / \mathrm{s}$. In Fig. 9, we plot $\mathrm{v}(L, t)$ vs $t$ from Eqs. (48) and (49). Because of the maximally dissipative kinetics, the particle velocity following the first wave is always $\mathrm{v}^{+}=c_{0} \gamma_{m}=1.170 \mathrm{~km} / \mathrm{s}$ in the two-wave cases; the values of particle velocity $\mathrm{V}^{\prime}$ following the phase boundary are those listed in Table III. Only those results predicted by the model for the four shots corresponding to impactor velocities $V=2.603,3.120,3.470$, and $3.900 \mathrm{~km} / \mathrm{s}$ are shown in Fig. 9, which is to be compared with the ex-

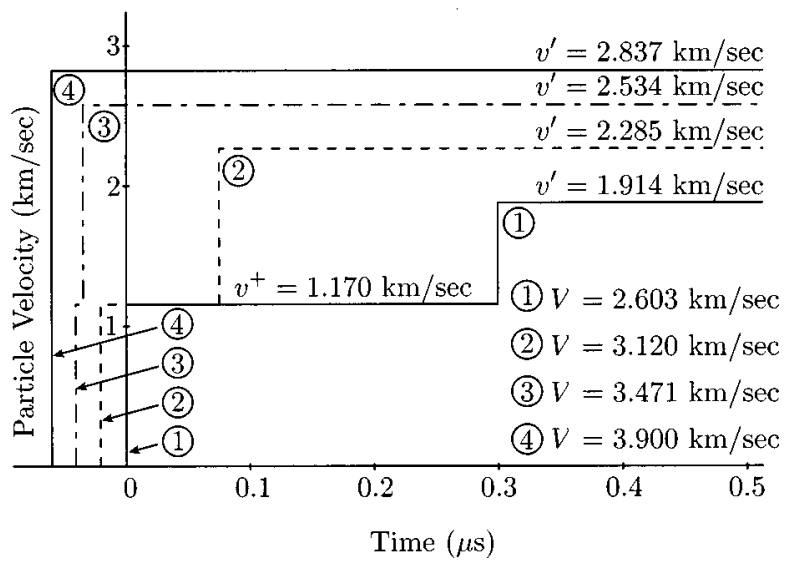

FIG. 9. Particle velocity time histories as predicted by the model for four of the impactor velocities $V$ used in the experiments in Ref. 5 .

perimental results shown in Fig. 1. As in Fig. 1 (reproduced from Ref. 5), the four curves in Fig. 9 have been staggered horizontally for clarity. Times between arrivals of the shock and the phase boundary in the two-wave cases were computed by choosing for the undeformed thickness $L$ of the target the specimen thickness for each experimental shot; these thicknesses are reported in Table I of Ref. 5.

For maximally dissipative kinetics, the "nucleation stress" $\sigma_{\text {nuc }}$ at which the phase transition first occurs is that associated with the local minimum in the stress-strain curve of Fig. 3. Also, the strain behind the phase boundary when the second wave just overtakes the first in the two-wave solution is that for which the Lagrangian phase boundary velocity $\dot{s}$ has just increased to the value $c_{0}$ of the speed of the front-running LPP shock wave. It follows from the geometric interpretation of Eq. (6) that this "overdrive strain" is $\gamma$ $=-\gamma_{*}$ (Fig. 3). The corresponding stress $\sigma_{\text {over }}$ is that at which the phase boundary is first overdriven. One finds that these special stresses are given by

$$
\begin{gathered}
\sigma_{\text {nuc }}=-\mu_{0} \gamma_{m}=-\rho c_{0}^{2} \gamma_{m}=-18.4 \mathrm{GPa}, \\
\sigma_{\text {over }}=-\mu_{0} \gamma_{*}=-\rho c_{0}^{2} \gamma_{*}=-40.0 \mathrm{GPa} .
\end{gathered}
$$

In Ref. 5, the "transition onset pressure'" is given as 19.6 $\pm 0.7 \mathrm{GPa}$, and it is reported that the phase transition is overdriven at pressures above $40 \mathrm{GPa}$.

At the values $\dot{s}=c_{0}$, the two relations (39) and (40) relating impactor velocity to Lagrangian phase boundary speed deliver the same value of $V$. This is the impactor velocity $V_{\text {over }}$ at the threshold of overdriven response; it is given by

$$
V_{\mathrm{over}}=\left(1+\frac{1}{\Omega}\right) \gamma_{*} c_{0}=3.490 \mathrm{~km} / \mathrm{s} .
$$

This is consistent with the experimental data in Table III

Finally, the quasistatic transformation strain $\gamma_{\text {trans }}$ of Eq. (12) for the model is

$$
\gamma_{\mathrm{rans}}=\gamma_{T}-\left(1-\mu_{0} / \mu_{1}\right) \gamma_{m}=0.181
$$


${ }^{1}$ R. Abeyaratne and J. K. Knowles, J. Mech. Phys. Solids 41, 541 (1993).

${ }^{2}$ R. Abeyaratne and J. K. Knowles, Philos. Trans. R. Soc. London, Ser. A 355, 843 (1997).

${ }^{3}$ P. Rosakis and J. K. Knowles, J. Mech. Phys. Solids 45, 2055 (1997).

${ }^{4}$ J. K. Knowles, Comput. Mech. 22, 429 (1999).

${ }^{5}$ D. J. Erskine and W. J. Nellis, J. Appl. Phys. 71, 4882 (1992).

${ }^{6}$ J. C. Escobar and R. J. Clifton, Mater. Sci. Eng., A 170, 125 (1993).

${ }^{7}$ J. C. Escobar and R. J. Clifton, Proc. SPIE 2427, 186 (1995).

${ }^{8}$ R. Abeyaratne and J. K. Knowles, SIAM (Soc. Ind. Appl. Math.) J. Appl. Math. 51, 1205 (1991).
${ }^{9}$ R. Abeyaratne and J. K. Knowles, Q. Appl. Math. 50, 149 (1992).

${ }^{10}$ J. R. Rice, J. Appl. Mech. 37, 728 (1970).

${ }^{11}$ J. Lubliner, Acta Mech. 52, 225 (1984).

${ }^{12}$ R. Abeyaratne, C. Chu, and R. D. James, Philos. Mag. A 73, 457 (1996).

${ }^{13}$ C. Chu, Ph.D. dissertation, University of Minnesota, Minneapolis, MN, 1993.

${ }^{14}$ R. Abeyaratne and J. K. Knowles, Acta Mater. 45, 1671 (1997).

${ }^{15}$ S. P. Marsh, LASL Shock Hugoniot Data (University of California Press, Berkeley, 1980) 